\title{
CONSERVATION DES ÉCHANTILLONS DE LAIT EN VUE DE LEUR ANALYSE ( $(1)$
}

\author{
par
}

\section{VOIRET}

Directeur du Laboratoire Municipal de Lyon

La bonne conservation des échantillons de lait est certainement le facteur le plus important pour leur analyse correcte. Tous les intéressés connaissent les avantages et les inconvénients du bichromate, le seul conservateur actuellement utilisé pour les prélèvements officiels : à l'actif, commodité d'emploi, bonne conservation des échantillons pendant un à deux mois, permettant la première analyse dans des conditions satisfaisantes.

Au passif, apport de matières minérales et d'alcali, gênant pour certaines phases de l'analyse, réduction et disparition progressive du conservateur qui laisse alors le champ libre aux coagulations et fermentations ultérieures, tannage de la caséine par les sels de chrome, lui donnant une structure granulaire difficile à résorber même par des moyens mécaniques et rendant les prélèvements à la pipette plus incertains, tous inconvénients qui rendent souvent aléatoires des contre-expertises ultérieures.

Nous avons donc cherché à définir une méthode de conservation qui n'aurait pas ces inconvénients et assurerait néanmoins une conservation illimitée du lait sous sa forme, son homogénéité et sa viscosité initiale. L'expérience montre que seule l'élimination totale des germes qu'il eontient permet ce résultat. Nous avons done recherché les moyens pratiques d'y parvenir.

La stérilisation d'un milieu peut s'obtenir de différentes façons :

\section{$1^{\text {. }}$ Action d'antiseptiques chimiques}

L'expérience montre que si certaines espèces microbiennes ne résistent pas à l'action d'antiseptiques courants tels phénols ou crésols, acide salicylique, etc., les ferments lactiques, par contre, se montrent extrêmement résistants, les antiseptiques ne faisant que retarder la fermentation; la coagulation à la température ordinaire se produit au bout de trois ou six jours. On aboutirait alors à l'inverse du résultat cherché, c'est-à-dire, que le lait arriverait régulièrement coagulé au laboratoire officiel.

Seuls des antiseptiques extrêmement énergiques comme les cyanures du cuivre ou de mercure arrivent à détruire les ferments lactiques; leur emploi toutefois, pour des raisons évidentes, ne peut être envisagé.

(1) Annales des falsifications et des fraudes, 1951, 511-513, 284. 
Un antiseptique mis sur le marché depuis peu, semble doué d'un pouvoir bactéricide important et semblerait donner des résultats intéressants à dose suffisante. Il est du type ammonium quaternaire à cation actif. Il a toutefois des inconvénients rédhibitoires :

Il contient du chlore ionisable qui fausserait le dosage des chlorures.

Il n'a ni odeur, ni couleur, ni réaction particulière, ni méthode de dosage suffisamment sensible qui permette d'en déceler la présence et la quantité.

\section{Conservation par action physico-chimique}

L'expérience montre que les microorganismes ne peuvent se développer que dans des conditions physico-chimiques assez précises et limitées.

$1^{\circ} p \mathrm{H}$. Si l'on abaisse le $p \mathrm{H}$ aux environs de 2 on arrête pratiquement les fermentations. Toutefois, l'addition en proportion bien définie d'un acide fort à l'échantillon ne serait pas pratique ;

$2^{\circ} r \mathrm{H}$. L'expérience montre que l'activité microbienne est maxima à une valeur assez bien définie $\mathrm{du} r \mathrm{H}$ et se poursuit à des vitesses variables dans une zone plus ou moins large selon les. espèces, de part et d'autre de cette valeur optima. Si done, on fixe le $r \mathrm{H}$ de l'échantillon en dehors de cette zone, on pourrait espérer a priori, une bonne conservation du lait.

Nous avions donc espéré qu'en fixant le $r \mathrm{H}$ à une valeur assez haute ou basse, on pourrait inhiber complètement toute activité microbienne.

Le bichromate d'ailleurs agit dans ce sens pour une part du moins. Le $r \mathrm{H}$ de lait bichromaté à la dose habituelle est de l'ordre de 28 à 29 , c'est-à-dire en dehors des zones d'activité maxima des germes principaux.

-Afin de préciser le comportement des ferments lactiques en fonction du $r \mathrm{H}$, nous avons étudié les variations $\mathrm{du} r \mathrm{H}$ dans divers laits en évolution normale ou dans certains milieux de cultures artificiels pour ferments lactiques.

L'expérience montre que la fermentation lactique s'accompagne de formation de produits à caractère réducteur et qui créeront dans le lait des $r \mathrm{H}$ extrêmement bas, de l'ordre de 4 à 6 .

Il paraît done vain, soit de prétendre utiliser eomme conservateur un oxydant qui ne soit pas peu à peu réduit, soit de penser trouver un élément réducteur d'emploi pratique, susceptible d'arrêter l'évolution de ferments lactiques.

Effectivement, les divers éléments que nous avons expérimentés, quinone (pour les oxydants), hydroquinone, sulfures, 
diphenylamine, soufre en solution dans $\mathrm{Cs}^{2}$ (qui donne de l'hydrogène sulfuré), tous ne font que retarder l'acidification au même titre que les antiseptiques signalés plus haut.

\section{$3^{\circ}$ Méthodes physiques}

Parmi les agents physiques stérilisants, la chaleur est, semble-t-il, le plus accessible. Nous avons cherché à définir une méthode de stérilisation compatible avec les possibilités matérielles limitées dont peut disposer la Répression des Fraudes au stade du prélèvement.

A cause de ces possibilités limitées, nous excluons, bien entendus la stérilisation à l'autoclave. De même, nous excluons les procédés de stérilisation à basse température par tyndallisation, trop délicats à appliquer pour être sûrs.

Nous nous sommes arrêtés, comme processus le plus pratique, au simple chauffage de l'échantillon à basse température, entre 55 et $70^{\circ}$, en présence d'un antiseptique. L'expérience montre, en effet, que l'action combinée de la chaleur et de l'antiseptique pendant une heure environ suffit pour provoquer une stérilisation complète. Cette opération ne nécessite aucune précaution spéciale; il suffit de placer l'échantillon prélevé comme à l'habitude dans une étuve réglée vers $65-70^{\circ}$ et de l'y maintenir un temps suffisant pour un chauffage homogène.

\section{Choix de l'antiseptique.}

Nous préconisons un antiseptique organique qui ne puisse gêner lors de l'examen des cendres. L'acide salicylique en solution amylique saturée nous 'paraît le plus indiqué; il a l'avantage de ralentir suffisamment la fermentation lactique pour laisser un délai de quarante-huit heures au moins sans acidification notable, qui laisse aux agents exécutant le prélèvement toute latitude pour effectuer normalement leurs opérations. Tout autre antiseptique analogue solide ou liquide peut toujours convenir. La dose suffisante peut être faible, $0 \mathrm{gr}$. 5 à $1 \mathrm{gr}$. par litre, soit 1 à $2 \mathrm{~cm}^{3}$ de solution saturée à $10 \%$ environ pour $200 \mathrm{~cm}^{3}$ de lait; (l'acide salicylique solide est difficile à utiliser, provoquant un caillot qui englobe l'antiseptique). Nous avons obtenu également de bons résultats avec la naphtaline dont l'emploi est plus commode; l'addition d'alcool amylique est toujours intéressant. Il améliore les contacts et facilite la ré-émulsion ultérieure de la crème; son pouvoir antiseptique est notable, il évite le barattage du beurre à la saison chaude.

\section{Matériel.}

10 Flaconnage de prélèvement constitué de préférence par des 
flacons fermés par capsule vissée-de préférence au bouchon, de façon à résister à une légère pression intérieure et assurer une bonne étanchéité. Un joint caoutchouc est préférable;

$2^{\circ}$ Etuve ou armoire chauffante réglée à $70^{\circ}$ environ par bilame ou tout autre dispositif; de dimension convenable pour le nombre de flacons à traiter.

\section{Mlode opératoire.}

Les flacons additionnés d'antiseptique lors du prélèvement sont simplement maintenus dans l'étuve de six à douze heures pour permettre à coup sûr l'égalisation de température et la stérilisation.

\section{Conclusion}

Les échantillons ainsi traités se conservent indéfiniment sans augmentation de l'acidité, ni de la viscosité. Leur analyse pour tous les éléments s'effectue dans les mêmes conditions que celles d'un lait frais. Les causes principales de controverse en cas de contreexpertise, liées surtout à l'état de conservation de l'échantillon, se trouvent éliminées. Aucune difficulté ne subsiste pour la recherche des carbonates éventuellement ajoutés.

Aucune difficulté particulière pour les agents effectuant les prélèvements, l'antiseptique étant contenu dans un flacon comptegoutte ; la quantité ajoutée étant dans une certaine mesure indifférente. La méthode nécessite simplement l'achat, dans chaque préfecture, d'une étuve électrique à régulateur valant quelques dizaines de milliers de francs seulement et dont le réglage et l'emploi ne présentent aucune difficulté spéciale.

\section{BIBLIOGRAPHIE ANALYTIQUE}

\section{LES LIVRES}

Dornic (P.). - Le contrôle pratique et industriel du lait.

1 volume broché de 142 pages, $11 \times 18,22$ fig. $6^{\text {e }}$ édition mise à jour par André Chollet. J. B. Baillière et Fils, éditeurs, 19, rue Hautefeuille, Paris $\left(6^{\circ}\right), 1951$. Prix : 350 franes.

En mettant à jour ce livre dont la première édition remonte à 1893 , A. Chollet déclare dans sa préface qu'il a désiré présenter un ouvrage élémentaire destiné, avant tout, aux industriels laitiers et aux élèves des écoles saisonnières de laiterie. Volontairement, ont été ici retenues les méthodes les plus simples et les plus généralement employées.

Après avoir rappelé les buts du contrôle et donné quelques généralités sur le matériel d'analyse, le chapitre premier traite de la composition du lait et de ses variations. 